\title{
Characterization of NIS neutron irradiation facility for calibration and metrological application
}

\author{
Ahmed R. El-Sersy ${ }^{1,}$, Eman A. Sayed ${ }^{1}$, Salwa H. Nagib ${ }^{1}$, Reham Hamdy Bakr ${ }^{1}$, and Randa M.M. Mahmoud ${ }^{2}$ \\ ${ }^{1}$ Ionizing Radiation Metrology Lab, National Institute of Standard, Giza, Egypt \\ ${ }^{2}$ Nuclear and Radiological Regulatorty Authority, Nasr City, Cairo, Egypt
}

Received: 24 September 2020 / Accepted: 10 March 2021

\begin{abstract}
In this study, the Neutron Irradiation Facility (NIF) of the National Institute of Standards (NIS) was characterized for metrological applications to improve the accuracy of the calibration process. The NIS neutron irradiation facility consists of a $5 \mathrm{Ci} \mathrm{Am}-\mathrm{Be}$ and $0.1 \mu \mathrm{g}$ Cf-252 sources. The flux and dose rate of the Am-Be source was calculated by using MCNP5 code simulation at different distances from the source. The dose rate delivered by the source was determined using NM2-neutron monitor at different source-to-detector distances. A comparison between the measured and the calculated dose rate was performed and the deviation between them was explained in the skeletal arrangement of room scattering contribution. A shadow cone was designed and constructed to determine the scattering contribution at different source-to-detector distances. The optimum source-distance used for calibration was specified. It was found that the Am-Be calculated flux vary with distances from about $10^{7}-10^{4}\left(\mathrm{n} / \mathrm{cm}^{2} \cdot \mathrm{S}^{-1}\right)$. The measured and the calculated dose rates were in agreement up to $150 \mathrm{~cm}$ distance from the source center after which the measured dose was greater than that calculated. The determined neutron scattering calculated from the measured-to-calculate dose ratio increased from $7 \%$ to $25 \%$ with increased distances from 150 to $300 \mathrm{~cm}$. Moreover, the standard dose used in the calibration should be measured by a standard neutron monitor at each distance due to the higher value of the room scattering contribution where the optimum distance for calibration was $150 \mathrm{~cm}$. The combined uncertainty of the measured neutron dose was $4.04 \%$.
\end{abstract}

Keywords: Ionizing radiation / neutron scattering / dosimetry

\section{Introduction}

The Ionizing Radiation Metrology Laboratory (IRML), in the Egyptian National Institute of Standard (NIS), is one of the IAEA Secondary Standard Dosimetry Laboratory (SSDL). The major SSDLs activities used to provide and disseminating traceability of the SI units to clients. Thus, it is imperative that the parameters affecting the traceability and uncertainties in its own irradiation facility have to be characterized thoroughly by reviewing irradiation setup and calibration procedures. The irradiation facility setup, the environmental conditions and the dosimetry system are the most substantial and important elements [1-5]. One of such activities is the calibration of neutron survey meters and neutron dosimeters.

Neutrons are produced from sources, generators and medical linear accelerators. Neutrons are indirectly

\footnotetext{
* Corresponding author: aelsersy@yahoo.com
}

ionizing radiation; they must react with some other medium to produce a primary ionizing particle. Different nuclear reactions should be used depending on the energy of the neutrons that need to be measured. Some of the basic neutron detection reactions used in health physics instrumentation are ${ }^{10} \mathrm{~B}(\mathrm{n} ; \alpha){ }^{7} \mathrm{Li}$, elastic scattering of high-energy neutrons by hydrogen atoms, nuclear fission and neutron activation. In the neutron irradiation facility, it's very important to study the room design, scattering contribution, and air attenuation because they use different distances through the calibration processes. The room in-scattering measurements are performed using the shadow cone method [6-11].

This work aimed to complete setting up of NIS neutron irradiation facility for metrological application according to the international regulations. Moreover, to characterize the neutron irradiation facility for calibration and dosimetry purposes and to study the variation of the dose delivered by Am-Be source with distances and with considering the room spread out. This work also aimed 
to calculate the combined uncertainty in neutron dose at an arbitrary distance from the neutron source and get the NIF for intercomparison and meteorological applications.

\section{Experimental and methodology}

The source used in the NIS neutron facility is a $0.185 \mathrm{GBq}$ Am-Be produced by Amersham [12]. The shield of this installation is composed of $45 \%$ hydrogenous material (asphalt), 5\% borax and 50\% sand which is collected in a wooden box of dimension $120 \times 120 \times 140 \mathrm{~cm}^{3}$ where the background is smaller than $1 \mu \mathrm{Sv} / \mathrm{h}$ on the surface of the boxwood. The box is in the irradiation room dimension of $4 \mathrm{~m}$ width, $4 \mathrm{~m}$ height, and $10 \mathrm{~m}$ length in the basement. The room is made of a forced concrete wall with a thickness of $80 \mathrm{~cm}$. The source motioned is controlled electronically outside of the irradiation room with the assistance of some motosr. Neutron doses were measured by a NM2 neutron monitor calibrated at the PTB [13]. A shadow cone is designed and constructed pyramidal in shape with a small bias of $5 \times 10 \mathrm{~cm}^{2}$ and a big base of $22 \times 22 \mathrm{~cm}^{2}$ and a thickness of $50 \mathrm{~cm}$ made of borates wax. The borated wax used as a shielding material for the used neutron source is smaller than $50 \mathrm{~cm}$ [10]. The shadow cone was constructed in such a way that the penetrated dose is within the uncertainty of the measured dose.

Figures $1 \mathrm{a}$ and $1 \mathrm{~b}$ shows the neutron irradiation facility used in this study (a) and the photograph of the irradiation facility (b).

The structure of the Am-Be neutron source used in this study was simulated by using MCNP5 [14,15], according to the Amersham catalogue [12]. The neutron source is a cylindrical capsule with $3 \mathrm{~cm}$ diameter and $6 \mathrm{~cm}$ height. The source is surrounded by a holder made of Poly-methyl methacrylate (PMMA) with $1 \mathrm{~cm}$ thickness. The dose rate was estimated using MCNP5 at different distances from the source center. The flux was calculated utilizing the point detector technique and F5 tally. The neutron dose (D) in this study is calculated using the relation:

$$
\mathbf{D}=\mathbf{h}^{*} \Phi
$$

where $\Phi$ is the neutron flux $\left(\mathrm{S}^{-1}\right)$ and $\mathrm{h}$ is flux to dose conversion factor obtained from the literature with a value of $391 \mathrm{pSv} / \mathrm{cm}^{2}$ [16]. Gamma photon flux to dose conversion factor as reported ICRP-21 [16] using dose function card DFn and dose energy card Den. A flux of $10^{6}$ Neutrons and $\gamma$-photons were used to simulate the transport of neutrons, $\gamma$-photons and accumulate neutrons, $\gamma$ tallies.

\section{Results and discussion}

\subsection{Neutron doses delivered by source}

The source used was $0.185 \mathrm{GBq}$ Am-Be in a capsule with $3 \mathrm{~cm}$ diameter and $6 \mathrm{~cm}$ length. Using MCNP5 code, the neutron flux free in air at a certain level was calculated at different distances from the source center, and represented in Figure 2. From this figure, one notices that the flux decreased $20 \%$ of its original value with a distance increases to $50 \mathrm{~cm}$ and then slightly decreases with distance up to $300 \mathrm{~cm}$.

Using the flux-dose conversion factor [16], the neutron dose rate was calculated by MCNP5 and measured by NM2 neutron monitor at different source-to-detector distances and represented in Figure 3. From Figure 3, it is obvious that the measured dose rate values are slightly smaller than those calculated up to distance of $150 \mathrm{~cm}$ and then the measured dose rate was increased more than that calculated with distance increases from 150 to $300 \mathrm{~cm}$.

This may be attributed to the in and out neutron scattering contribution. At short distances, the out scattering is higher than the inner one, hence the measured dose is smaller than the calculated one while at a distance greater than 150 the in-scattering factor is increased and so the values of the measured doses are greater than that of the calculated ones. To confirm the obtained conclusion, the ratio of the measured dose rate to the calculated ones is calculated from the above figure and is represented with the different source- to- detector distance in Figure 4. Figure 4 indicated that with distance less than $150 \mathrm{~cm}$ the measured dose is smaller than the calculated ones, but with distance above $150 \mathrm{~cm}$ the measured dose rate is greater than the calculated due to the contribution of the in scattering than the out scattering values.

\subsection{Determination of neutron scattering}

The constructed pyramidal shadow cone was used to assure the protection of the monitor against primary beam, and measured only the scattering one (see Fig. 1).

The dose delivered by the source was measured with and without the shadow cone at different source-todetector distances. The percentage ratio of measured with cone and that without is represented in Figure 5.

From this figure, it can be noticed that the ratio of neutron scattering increased from $7.5 \%$ to $25 \%$ with a distance increases from 50 to $300 \mathrm{~cm}$. This may be attributed to the growth of the beam divergence where the neutron scattering increases with increasing the distance.

\subsection{Effect of monitor geometry}

It is well known that the scattering measurements depend upon the geometry of the neutron monitor probe. To study the effect of the probe shape on the scattering measurements, two common types of neutron monitors with different geometry such as cylindrical and semi-spherical were used. The neutron scattering by the two monitors was measured at different source-monitor distance and was represented in Figure 6. From this figure, one notices that the neutron scattering measured by different probe shapes was in agreement with each other and the variation was in the range of experimental uncertainty. 


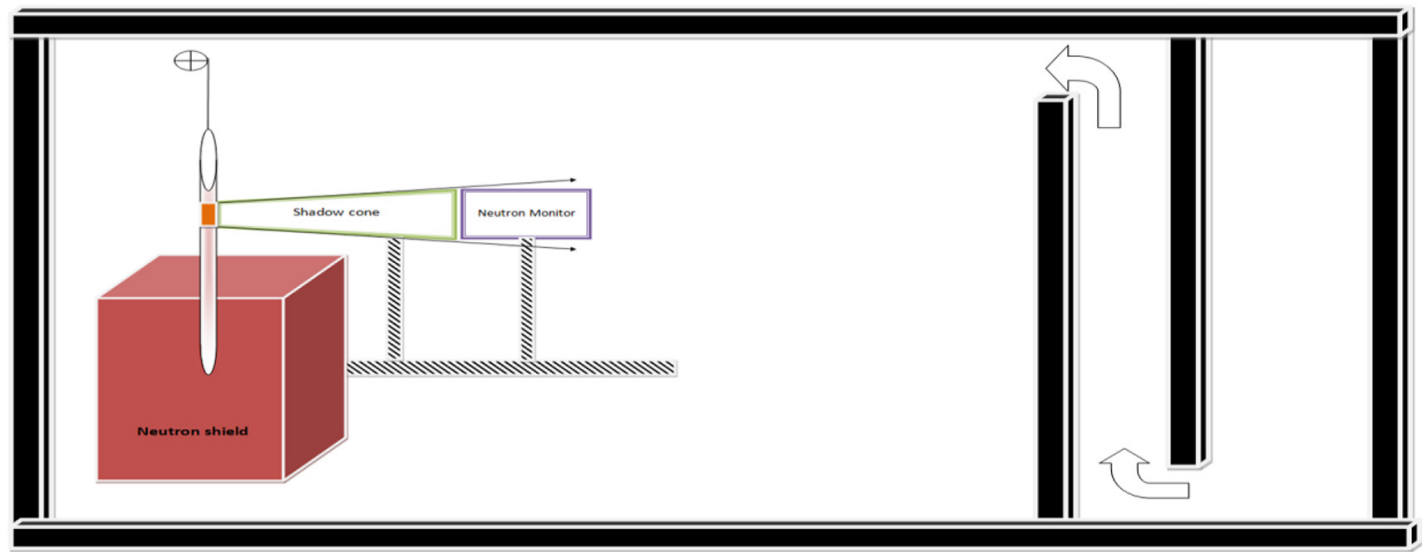

(a)

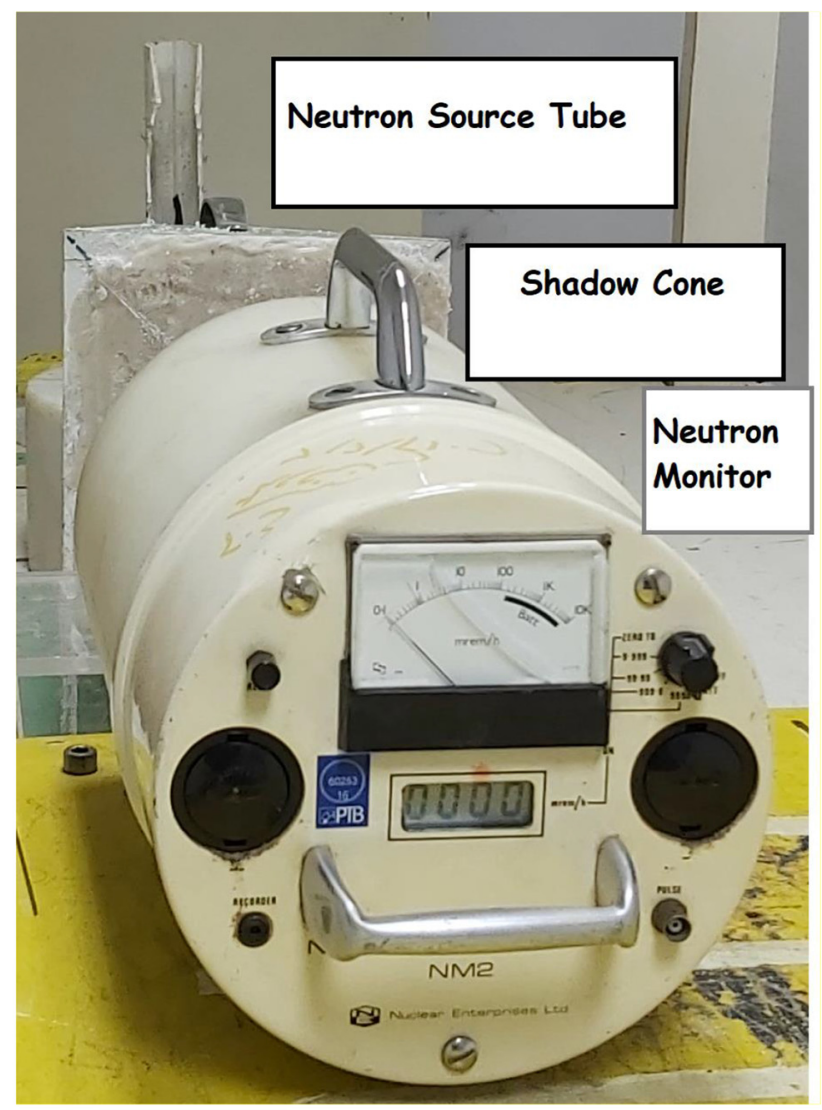

(b)

Fig. 1. Schematic diagram of the neutron irradiation facility (a) and its photograph (b).

\subsection{Uncertainty of the neutron doses}

The uncertainty due to independent parameters associated with the measured neutron doses may have a statistical component (type A) or regular ones (type B) and the total combined uncertainty was calculated from equation (2) $[17,18]$.

$$
\mathrm{U}_{\mathrm{C}}=\sqrt{\sum \mathrm{U}_{\mathrm{A}}^{2}+\sum \mathrm{U}_{\mathrm{B}}^{2}}
$$

where $\mathrm{U}_{\mathrm{A}}$ is the combined uncertainty from type $\mathrm{A}$ sources and $U_{B}$ is the combined uncertainty from type B sources.

Combined uncertainty is determined considereding the different sources as follows:

- The Repeatability and the reproducibility Statistical uncertainty of neutron dose measurements $(20$ and 5 readings for repeatability and reproducibility, respectively) were taken and the standard deviation was used in their calculations (type A). 


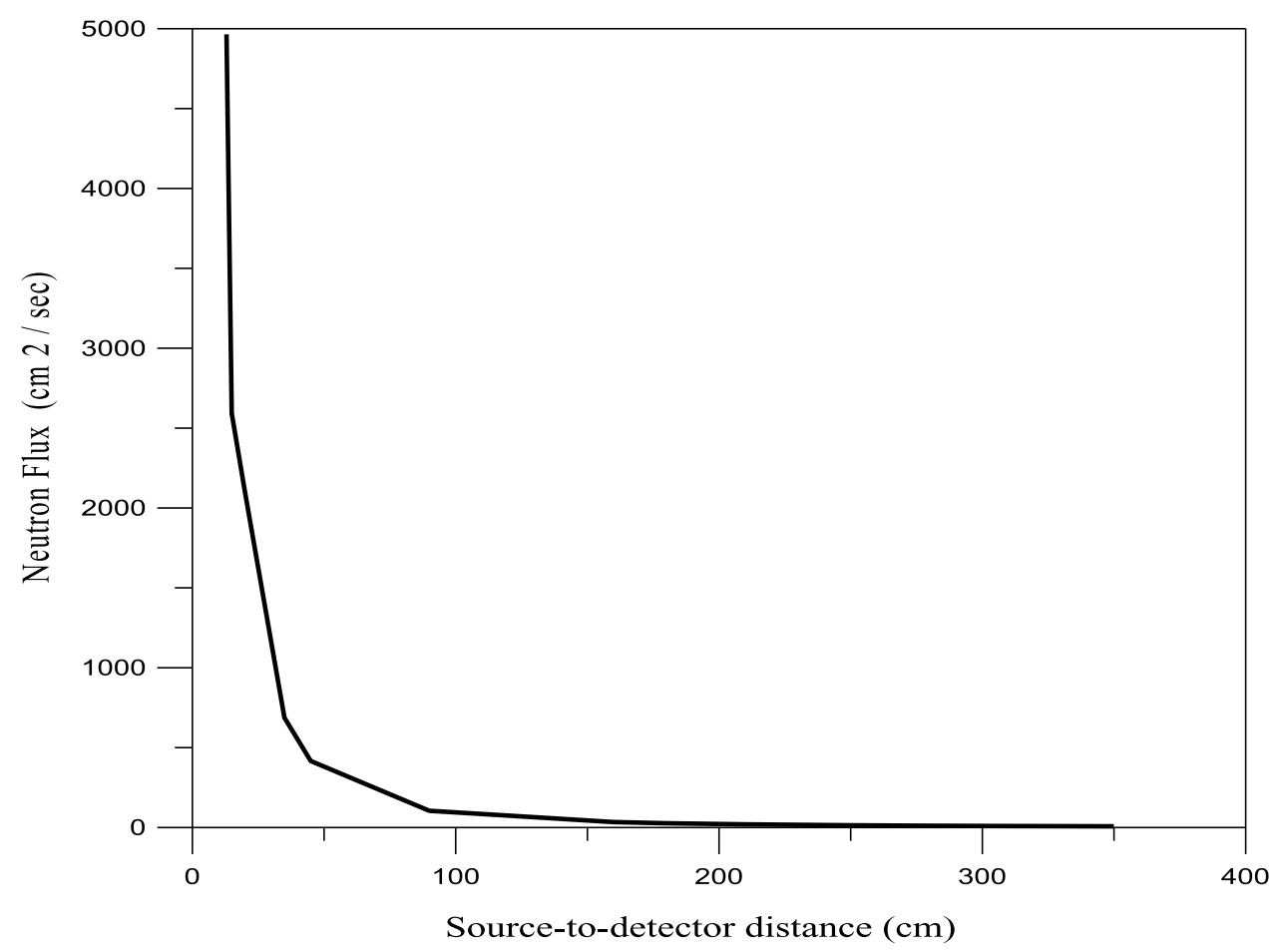

Fig. 2. The calculated neutron flux by MCNP5 at different distances from the source center.

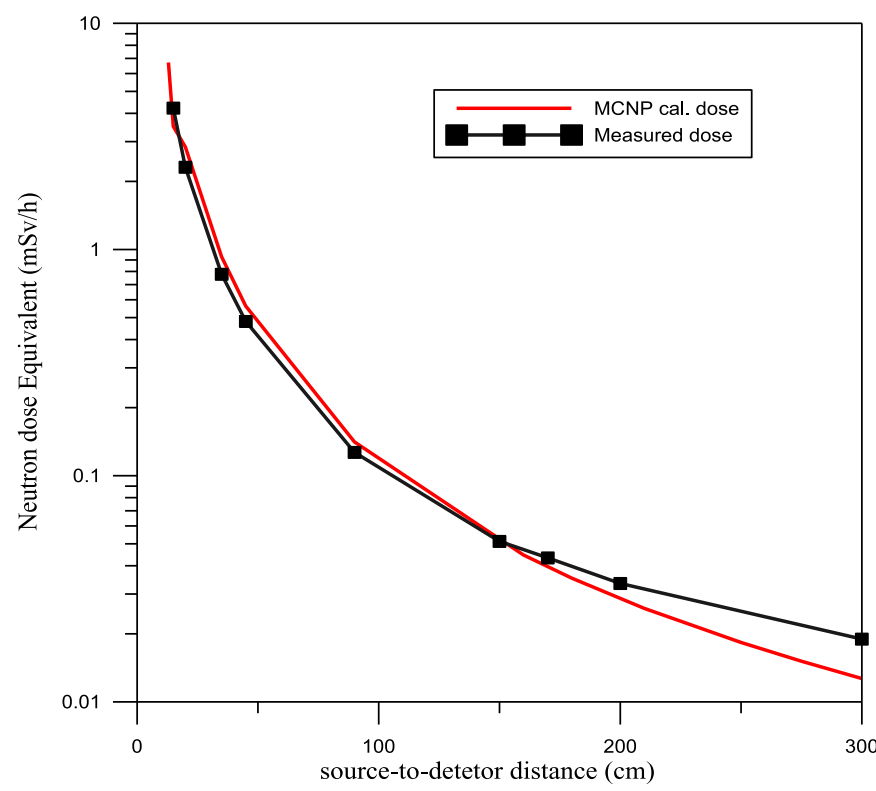

Fig. 3. A comparison between the calculated and measured neutron dose rate at different source-to-detector distances.

- Neutron monitor stability was estimated using the different reading of the check source with a constant emission rate and the standard deviation was used as a type A.

- The linearity of dose that related to the response of the used neutron monitor to different neutron energies which has a value of $2 \%$ type $B$.

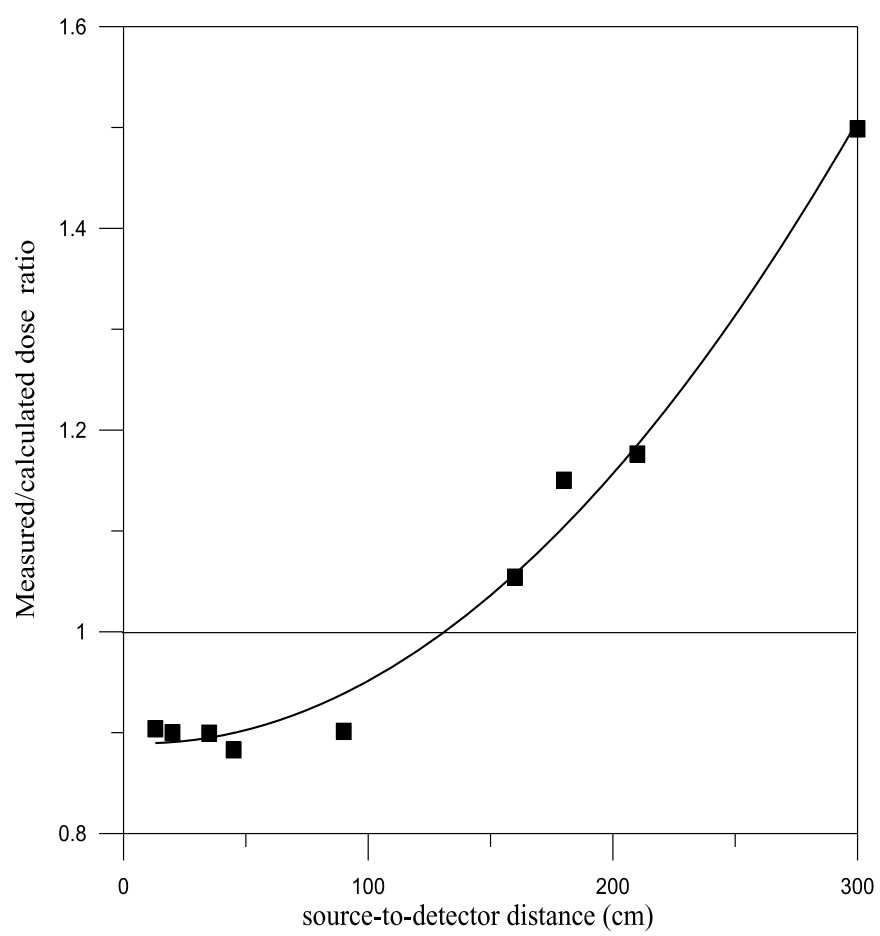

Fig. 4. Measured-to-calculated dose ratio with different sourceto-detector distances.

- Resolution of the reading of the neutron monitor with has a value of $1.2 \%$ type $B$.

- Monitor position at the distance used for dosimetry. This source of uncertainty has type A from the repeatability of 


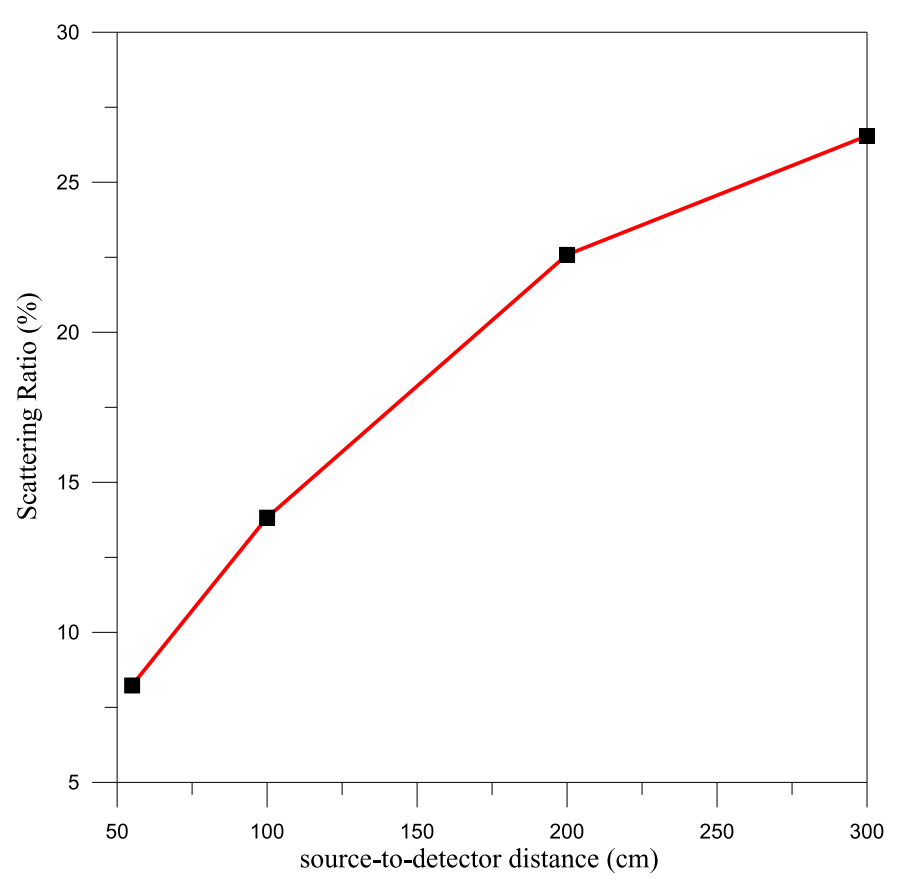

Fig. 5. The neutron scattering ratios with source to detector distances.

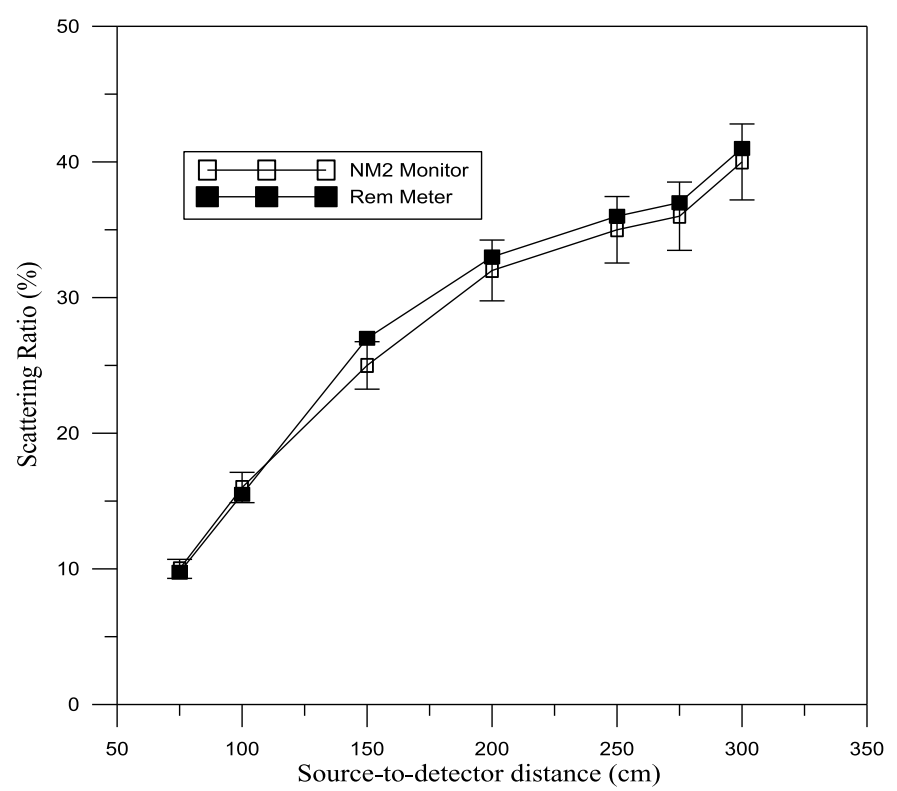

Fig. 6. Neutron scattering ratio as measured with different probe shape with different distances.

distance measurements and type B from the calibration factor of the distance meter used where the uncertainty of distance is converted to neutron dose by the aid of inverse square law.

- The neutron scattering: The uncertainty of the room scattering was measured by the shadow cone, however the uncertainity of the air acattering was taken from the ISO No. 8529-2:2000 [19] which has a value of 1 type A and $1.5 \%$ type.
Table 1. The uncertainty budget in the measured dose.

\begin{tabular}{lll}
\hline Source of uncertainty & $\begin{array}{l}\text { Type A } \\
\text { value }(\%)\end{array}$ & $\begin{array}{l}\text { Type B } \\
\text { value (\%) }\end{array}$ \\
\hline Repeatability reproducibility & 2 & - \\
Neutron Monitor stability & 1.5 & - \\
Linearity with dose & - & 2 \\
Reading resolution & - & 1.2 \\
Monitor position & 0.1 & 1 \\
Neutron scattering & 1 & 1.5 \\
Air attenuation & 0 & 0.6 \\
Uncertainty of each type & 7.26 & 9.05 \\
\hline
\end{tabular}

$\mathrm{Uc}=4.04 \%$. U expanded is $4.04 \%$ with coverage factor 1and confidence level of $65 \%$.

- The air attenuation. The uncertainty of the air attenuation is due to the decrement in the neutron dose through the source-monitor distance considering the attenuation coefficient of Am-Be neutron in the air which has a value of $890 \times 10^{-7} \mathrm{~cm}^{-1}$.

The distribution of the types $\mathrm{B}$ components are considering as a rectangular distribution where their final values are in the above budget [18] (Tab. 1).

\section{General discussion}

The neutron irradiation facility used in the standard laboratories should be constructed in such a way that the scattering contribution is minimized to be hardly contracted. If the neutron irradiation facility has a scattering component, it should be assessed at different source-todetector distances used for the calibration. In this work, the scattering component is determined firstly from the proportion of the measured to the calculated dose by MCNP5 code. A shadow cone was also utilized for quantitative measurements of neutron scattering from the ratio of neutron dose measurement with and without the shadow cone where the in-scattering has a value of $7.5 \%$ was obtained at a distance of $50 \mathrm{~cm}$ from the source and a value of $25 \%$ at a distance $300 \mathrm{~cm}$. This may be attributed to the increase of the beam divergence with source-todetector distance increases, the neutron scattering increases. The obtained scattering value of this study is in agreement with that obtained by Dantas et al. [1].

Different probe shapes of different common neutron monitors types of neutron monitors used to analyze the monitor geometry effect on the scattering measurements where an agreement between different monitor shapes was obtained. About eight sources of uncertainty were considered which extracted combined uncertainty of $4.04 \%$ in neutron dose at a distance of $200 \mathrm{~cm}$ from the origin. It is recommended from this study that the optimum distances used for calibration are between 100 to $200 \mathrm{~cm}$ in this neutron irradiation facility.

Grounded in the above assumption, it is recommended that during the utilization of this facility in the calibration purposes, the reference dose should be measured by a reference 
standard neutron dosimeter at each distance used for calibration to give the ability to measure both of the direct and the scattered neutron. Moreover, the probe geometry does not affect the dose determination in the standardization processes.

\section{Conclusion}

From the present work, it was concluded that the constructed NIF could cover a wide range of neutron doses from a few $\mu \mathrm{Sv} / \mathrm{h}$ to about $10 \mathrm{mSv} / \mathrm{h}$ by varying the source-to-dosimeter distance. The calculated neutron dose is in an agreement with the calculated one. Using the constructed shadow cone, the neutron scattering component at each irradiation distance was well calculated. Through the usage of this NIF, the standard neutron dose should be measured at each irradiation distance by a traceable neutron monitor for minimizing the uncertainty due to the neutron scattering. Different probe shape of the common neutron monitor has not affected the value of neutron scattering. The optimum calibration distance was found to be between 100 and $200 \mathrm{~cm}$ where uncertainty is minimized due to neutron scattering. Uncertainty of neutron dose was estimated and found to be $4.04 \%$ and distance-dependent.

\section{References}

1. M.L. Dantas, B. Mendes Freitas, C.L.P. Mauricio, K.C. de Souza Patrão, International Nuclear Atlantic Conference INAC Belo Horizonte, MG. Brazil. October 24-28 (2011)

2. M.P. Medeiros, J.J. Estrada, R. Gomes, S. Leite, R.F. Santos, C.F. Alves, W. Rebello, C. de Almeida, Congresso Brasileiro de Metrologia, Ouro Preto/MG, novembro de (2013)
3. B.M. Freitas, W.W. Pereira, K.C.S. Patrão, E.S. Fonseca, C.L.P. Mauricio, Radia. Prot. Dosimetry 161, 176-180 (2014)

4. S.I. Kim, B.H. Kim, J.L. Kim, J.I. Lee, Nucl. Eng. Technol. 47, 939-944 (2015)

5. M.A. Hassan, E. Esmat, A.R. El-Sersy, Development of 137Cs irradiation facility for metrological application, Int. J. Metrol. Qual. Eng. 8, 20 (2017)

6. A.R. El-Sersy, S.A. Eman, N.E. Khaled, Nucl. Inst. Meth. Phys. Res. 226, 345-350 (2004)

7. A.R. El-Sersy, Nucl. Inst. Meth. Phys. Res. 226, 234-238 (2010)

8. A.R. El-Sersy, S.A. Eman, Eur. Phys. J. A 226, 397-401 (2010)

9. N.E. Khaled, E.H. Ghanim, K. Shinashin, A.R. El-Sersy, Radiat. Eff. Defects Solids 169, 239-248 (2015)

10. A.R. El-Sersy, Int. J. Nucl. Energy Sci. Technol. 226, 97-106 (2016)

11. A.R. El-Sersy, N.E. Khaled, S.A. Eman, Nucl. Inst. Meth. Phys. Res. B: Beam 226, 443-448 (2004)

12. Amersham, The Radiochemical Center Technical Bulletin 77/8, Sources; Americi Beryllium (1977)

13. A.R. El-Sersy, S.A. Eman, M. Ezzat, R.A. Ali, Egypt. J. Biophys. 11, 15-27 (2015)

14. X-5 Monte Carlo Team, MCNP - A General Monte Carlo N-Particle Transport Code, Version 5. Los Alamos National Laboratory (2003)

15. R.M.M. Mahmoud, Design of a portable shield for neutron sources using MCNP computer code, Arab J. Nucl. Sci. Appl. 226, 1-8 (2018)

16. D.B. Pelowitz, MCNP5TM usere's Manual Los Alamos National Laboratory (2005)

17. IAEA Safety Standards Series No. SSR-6, Regulations for the Safe Transport of Radioactive Materials, Vienna (2012)

18. IAEA/TECDOC/1585, Measurement Uncertainty A Pratical Gide for Secondary Standard Dosimetry Laboratories (2008)

19. ISO No. 8529-2: Calibration fundamentals of radiation protection devices related to the basic quantities characterizing the radiation field (2000)

Cite this article as: Ahmed R. El-Sersy, Eman A. Sayed, Salwa H. Nagib, Reham Hamdy Bakr, Randa M.M. Mahmoud, Characterization of NIS neutron irradiation facility for calibration and metrological application, Int. J. Metrol. Qual. Eng. 12, 9 (2021) 